\title{
In-vehicle detection of targeted CAN bus attacks
}

\author{
Florian Fenzl \\ florian.fenzl@sit.fraunhofer.de \\ Fraunhofer Institute for Secure \\ Information Technology \\ Darmstadt, Germany
}

\author{
Roland Rieke \\ roland.rieke@sit.fraunhofer.de \\ Fraunhofer Institute for Secure \\ Information Technology \\ Darmstadt, Germany
}

\author{
Andreas Dominik \\ andreas.dominik@mni.thm.de \\ University of Applied Sciences \\ Mittelhessen \\ Giessen, Germany
}

\begin{abstract}
Most vehicles use the controller area network bus for communication between their components. Attackers who have already penetrated the in-vehicle network often utilize this bus in order to take control of safety-relevant components of the vehicle. Such targeted attack scenarios are often hard to detect by network intrusion detection systems because the specific payload is usually not contained within their training data sets. In this work, we describe an intrusion detection system that uses decision trees that have been modelled through genetic programming. We evaluate the advantages and disadvantages of this approach compared to artificial neural networks and rule-based approaches. For this, we model and simulate specific targeted attacks as well as several types of intrusions described in the literature. The results show that the genetic programming approach is well suited to identify intrusions with respect to complex relationships between sensor values which we consider important for the classification of specific targeted attacks. However, the system is less efficient for the classification of other types of attacks which are better identified by the alternative methods in our evaluation. Further research could thus consider hybrid approaches.
\end{abstract}

\section{CCS CONCEPTS}

- Security and privacy $\rightarrow$ Intrusion detection systems; • Computing methodologies $\rightarrow$ Machine learning.

\section{KEYWORDS}

Controller area network security; Intrusion detection; Anomaly detection; Machine learning; Automotive security; Security monitoring; Genetic Programming

\section{ACM Reference Format:}

Florian Fenzl, Roland Rieke, and Andreas Dominik. 2021. In-vehicle detection of targeted CAN bus attacks. In The 16th International Conference on Availability, Reliability and Security (ARES 2021), August 17-20, 2021, Vienna, Austria. ACM, New York, NY, USA, 7 pages. https://doi.org/10.1145/3465481. 3465755

\section{INTRODUCTION}

As technology standards improve, modern vehicles get smarter with each new model. Such smart cars are the preferred targets of

Permission to make digital or hard copies of part or all of this work for personal or classroom use is granted without fee provided that copies are not made or distributed for profit or commercial advantage and that copies bear this notice and the full citation on the first page. Copyrights for third-party components of this work must be honored

For all other uses, contact the owner/author(s).

ARES 2021, August 17-20, 2021, Vienna, Austria

(C) 2021 Copyright held by the owner/author(s).

ACM ISBN 978-1-4503-9051-4/21/08.

https://doi.org/10.1145/3465481.3465755 today's attacks on automotive systems [7]. Due to the longevity of vehicles in most cases a preventive security approach is not sufficient, therefore a continuous method of in-vehicle intrusion detection is required. Many attacks exploit certain weak points in the communication between the various electronic control units (ECU) and with external systems. The standard for the in-vehicle communication between ECUs is via the Controller Area Network (CAN) bus [6], which is highly vulnerable to cyber attacks [1]. The structure and semantics of the CAN bus payload is not standardized. It varies between the manufacturers and information about it is not available for open access. Thus, models for IDS created by hand require a high level of knowledge about the data and a significant amount of work to create. The behavior and constraints of CAN favor machine learning approaches [33] such as artificial neural networks (ANN) which rely only on the amount and quality of recorded data. However, ANN approaches are often limited in their portability by dependencies and a complex software environment. Most importantly, ANN-based IDS applications are rarely deployed [30] because a lack of insight into IDS results effectively prevents mitigation actions.

In our work, we consider decision trees trained through genetic programming (GP) which utilize only a set of basic operations and can easily be implemented. In addition, compared to other machine learning approaches (such as ANN), these trees are much easier to understand and optimize. They can be easily adapted to new intrusions and allow for dynamic rule generation. Through the selection of operators used by the GP algorithm, the focus of classification can be directed towards specific areas. For this purpose we have chosen the operators in such a way that the detection of correlations in separate data fields is supported. These advantages make GP an interesting research approach in the field of in-vehicle IDS, especially with regard to the observation of correlations between different data fields, which we consider important for the identification of targeted attacks.

The main contributions of this work are (i) an evaluation of the classification abilities for complex intrusions using GP decision trees as in-vehicle CAN bus IDS with the focus on message payload, (ii) an optimization through prior classification of the message payload, utilizing the field classification (FC) approach by Markovitz et al. [20], and, (iii) a comparison of the GP approach to other machine learning approaches, in particular sequential ANN, as well as the characteristic functions (CF) approach by Chevalier et al. [4]. The configuration of all approaches was determined with respect to a later implementation on low-performance edge devices within a vehicle. This is especially important for the design of the ANN.

The paper is organized as follows: First, we provide an overview on background and related work in Sect. 2. Section 3 introduces the CAN bus and the data sets used for the evaluation. Section 4 
describes the basics of GP, as well as the specifics of our method. Section 5 presents the results from GP and the compared approaches, while Sect. 6 discusses the results and Sect. 7 concludes this paper.

\section{RELATED WORK}

In 2010 Koscher et al. [15] analysed practical vulnerabilities on vehicles. Valasek and Miller [22] demonstrated real intrusions by inserting malicious messages via the CAN bus on vehicles such as the Ford Escape or Toyota Prius and later remotely on a 2014 feep Cherokee [23]. Studnia et al. [32] provided a short survey of attacks against ECUs. Payload-based approaches have been researched using a variety of machine-learning methods. Taylor et al. [34] utilized recurrent neural networks (RNN), precisely an Long Short-Term Memory (LSTM) approach as well as Gated Recurrent Units (GRU) to predict message sequences, while Song et al. [31] evaluated deep convolutional neural networks and Seo et al. [29] used generative adversarial nets for intrusion detection.

A different approach by Marchetti et al. [19] used a transition matrix for valid ID sequences. Chevalier et al. [4] proposed a characteristic functions approach focussed on the validity of payload values. The latter approach is further used in this work for comparison. Many of these approaches disregard correlations within the data and the context of the messages.

The usage of GP has proven efficient in other fields of research, such as brain-computer-interfacing [27], in finding correlations within a variety of unknown variables to build an effective and expandable model for classification. In network intrusion detection genetic algorithms have already been researched on several occasions [11]. Their general advantages and advantages for intrusion detection systems have been shown by Majeed et al. [18]. Application of genetic algorithms and GP in the context of CAN bus or general in-vehicle intrusion detection is rare, to the extent that we were unable to find any publications in this particular area. Crosbie et al. [5] and Bridges et al. [3] have utilized genetic algorithms in the field of network anomaly detection early on. Network intrusion detection using GP has been done by Lu and Traore [17] in a similar form to the approach of this work. Recently Cisco Technology patented an anomaly detection system for networks using GP [10].

\section{APPLICATION SCENARIO}

The aim of this work is to support early identification of intruders injecting malicious messages [22,26] or manipulating existing messages on the CAN bus in order to eventually take over safetyrelevant systems such as the brakes or motor control. The CAN bus [25] is a multicast-based communication protocol [6] that has become the standard protocol used in modern vehicles. The protocol was developed to be cost-effective, provide light protocol management and features for error detection and re-transmission.

There are several security concerns with the CAN bus. Physical access for malicious components can easily be gained through the on-board diagnostics (OBD) port [15, 22], remote access can be gained through insecurities of connected components such as the radio or remote diagnostic system [23]. The broadcasting of messages on the bus to every connected ECU can be used to snoop on communication, the priority handling using IDs results in a high vulnerability for denial of service (DoS) intrusions and there is no actual identification of messages [15]. Although the standardized automotive open system architecture (AUTOSAR) specifies how secure onboard communication (SecOC) can be used to check the authenticity of protocol data units, this method is not acceptable on classical CAN bus in many cases because of the high busload and subtle synchronisation problems [14, 28]. We have not found any SecOC elements within the log files that we used during our work. The priority of a message is determined by its identifier (ID). Messages are broadcast on the bus and every component determines whether the message is of interest by observing said IDs.

\begin{tabular}{r|c|c|c|c|c|c|c|c|}
\hline & Byte \\
\hline \multicolumn{2}{|c|}{8} & 8 & 8 & 8 & 8 & 8 & 8 & 8 \\
\hline \hline
\end{tabular}

\section{Figure 1: CAN bus frame payload alignments for ID $0 \times 5 d 7$}

Up to 8 bytes are reserved for the payload, whereby rarely only a single value is encoded in these fields, but several. The exact encoding and length of the different values is proprietary and differs from one manufacturer to another. Alignments and additional meta information are typically stored in data base CAN (DBC) files. In many published IDS approaches byte alignments (BA) are used for the evaluation of the data. This can distort the actual values and impede training and classification. Therefore, we utilize the FC approach by Markovitz et al. [20] to gain more accurate values compared to the byte-wise evaluation. In addition to this, we utilize alignment data from the CanZE open source project [8], which provides data base CAN files for a Renault ZOE from which our primary test log files were recorded. In Fig. 1 different exemplary alignments for the message bits with the respective numbers of bits per field, are shown. The FC row shows reverse engineered alignments, DBC shows alignments from CanZE. Each block represents a sensor or otherwise related value. Entries marked with a star are not used according to the given alignments. In these examples, the boundaries of the fields gained from the FC approach match the DBC alignments better than simple BA.

Test data collection. For our tests, we have recorded the CAN bus traffic of a Renault Zoe electric car. The data was recorded in an urban environment over the course of $\sim 550$ seconds, while driving up to $30 \mathrm{~km} / \mathrm{h}$, as well as standing still for short periods. About 1 million messages with 110 unique IDs were logged. The original data set contains no intrusions. We have evaluated all considered methods on the complete log file, but we will focus on two IDs for this work, namely, $0 \times 29 a$ with 26,836 recorded messages and $0 \times 5 \mathrm{~d} 7$ with 5,367 messages, for which we have designed specially targeted intrusion scenarios for the revolutions per minute (RPM) and speed values in the vehicle. The selection of these IDs and design of said targeted intrusions was influenced by Miller and Valasek [22] and existing open intrusion data sets, such as HCRL Car-hacking [12]. In order to cover a wide spectrum of CAN data from different vehicles, we also used a log file recorded from a Tesla Model X that we obtained from a collaboration with other researchers. This data set contains approx. 2.5 million messages with more than 100 different IDs from 25 minutes of recorded CAN traffic. 
Additionally, we utilized the HCRL Car-Hacking dataset [12], that has been used in several other CAN IDS approaches [29, 31, 35]. The HCRL data set contains 30 to 40 minutes of CAN traffic, with about 4 million messages in total. For our approach, we only evaluated the spoofing attacks on driving gear and RPM gauge from this set. In these attacks, only the messages for a single ID out of a total of approximately 25 IDs were attacked in each case. Therefore only messages with these IDs were evaluated in our tests.

Design of intrusion scenarios. We developed a set of intrusion scenarios based on the attack types reported in the literature. We composed a set of synthetic log files for these scenarios, ranging from simple DoS intrusions to complex manipulations which require whole ECUs to be compromised (see Table 1). The effects of the designed intrusions could not be tested on an actual test vehicle for safety reasons [36]. However, the impact of the U/SPEED intrusion, as well as potentially the U/RPM_LEFT intrusion described below can be estimated based on the work of Miller and Valasek [22].

The last three intrusions manipulate two sensor values directly and mask the actual values. Especially the U/SPEED intrusion is hard to detect because a successful detection requires the observation of subsequent messages. This intrusion is based on the speedometer intrusion on a Ford vehicle done by Miller and Valasek [22]. This intrusion is particularly difficult to classify for rule-based classifiers that only focus on the consistency of the value behavior. We have chosen a section of the log file in between two stops of the vehicle, where the data shows an acceleration to about $30 \mathrm{~km} / \mathrm{h}$ and set the velocity value to 0 . From the observation of a single message of the ID $0 \times 5 d 7$ alone, this is no abnormal behaviour. A classifier would need to detect the coherence between the speed value and another indicator. The payload of messages with this specific ID also contains the distance the vehicle has travelled. An intrusion can therefore be correctly classified when the velocity is zero, but the distance travelled changes. The training data set does not include any intentional examples of this intrusion, but only randomized messages. U/DoS and U/Fuzzy are not based on specific other publications, but are basic attack approaches in every security area. None of the shown intrusion scenarios where used in the training, with the exception of U/Fuzzy, where variations of the scenario on clean data are used to create the entire training log.

\section{GENETIC PROGRAMMING (GP) APPROACH}

Designed as an extension to genetic algorithms, GP is described as a systematic method to get a computer to automatically solve a problem [16]. This is done by transforming sets of given computer programs into generations of new programs by applying genetic operations like selection, mutation or crossover. Scoring of each program of a generation makes it possible to select only the best for further evaluation. Individual programs or genomes are expressed as syntax trees consisting of primitives and terminals $[9,16]$ taken from a predefined finite set. In the following, we will refer to these syntax trees as either programs or individuals.

Primitives in this case are either logical (AND, OR, NOT), relational $(\mathrm{LT} /<, E Q /==)$ or conditional (IF) operations, as well as a function that computes the absolute difference between two floating point values.
Terminals may either be a placeholder for a data field of the current message or previous message from the same ID, a randomized floating-point number between 0 and $2^{l}$, where $l$ is the maximum length of a messages field, or a Boolean constant True/False. Not every field of the message is necessarily used by the program.

The result of the evaluation of a message using such a model is a binary classification of the message, in the form of a simple Boolean value, telling if the message is an intrusion.

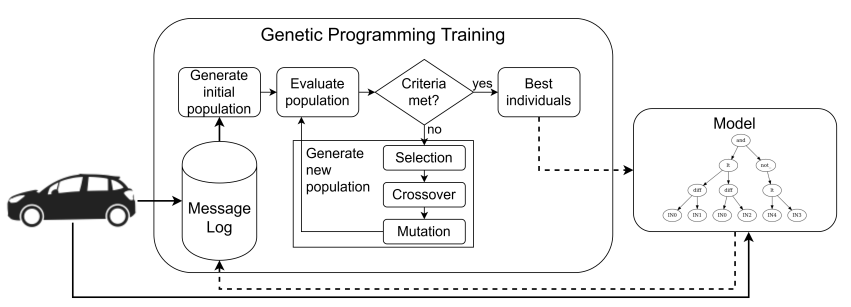

Figure 2: Structure of a Genetic Algorithm [13] and inclusion within the system

The training procedure of the GP algorithm, as well as the integration into a vehicle, is shown in Fig. 2. The training is initialized by generating a randomized population of 200 programs. Each primitive and terminal is chosen as a node randomly with equal probability, with each instruction being strongly typed [24]. Every individual of a generation will first be scored on their performance for the given training data. The method to determine the score referred to as fitness is critical to the success of the approach since it determines which of the individuals will be further evolved. In our approach fitness is derived from both the accuracy value of the classification on a clean, non-intruded log file, as well as the accuracy on the intruded training $\log \left(\right.$ fitness $=.75 *$ acc $_{\text {clean }}+.25 *$ acc $\left._{\text {train }}\right)$.

Through the priorisation of clean data classification, training is directed on the successful classification of valid data and the avoidance of false positives. After the fitness evaluation, the exit conditions of either a perfect accuracy or exceeding a threshold of 150 generations, are checked. The algorithm will then terminate, returning the 10 best individuals found over all generations. After fitness evaluation a tournament selection [21] is performed on sets of 10 individuals at a time in regard to their fitness value to create an offspring generation, where one individual program may even be selected multiple times by chance. In the next steps, each program in the offspring generation has a $50 \%$ chance to have a random sub-tree swapped with another random program from the same generation using single-point crossover and a $20 \%$ chance to have a sub-tree replaced by a newly generated tree for mutation. To remove obvious redundancies from the model, such as unambiguous conditional and logical operations on static Boolean values or a comparison operation on the same field, a minimization step is introduced. Our approach solely focuses on the evaluation message payload without consideration of frequency. Models are trained individually for each unique identifier within the CAN bus training data.

In order to evaluate the performance and recognition rate by models trained with GP, this approach is tested not only on bytealigned data, but also on data for which alignments were reverse engineered by FC [20]. Models generated by this approach are saved 
Table 1: Intrusion Scenarios

\begin{tabular}{ll}
\hline Attack & Description \\
\hline Urban & $\begin{array}{l}\text { A clean log file from the Renault ZOE without intrusions is used to test the operative readiness of the generated models. } \\
\text { Intrusion scenarios derived from this log are prefixed with 'U/'. }\end{array}$ \\
\hline U/Fuzzy, U/DoS & $\begin{array}{l}\text { A DoS type intruder, where the payload is randomized (U/Fuzzy) or set to zero (U/DoS). Over the course of 100s, a total } \\
\text { of 5,000 messages for each of the tested IDs was mixed into the clean log file. }\end{array}$ \\
\hline U/SPEED & $\begin{array}{l}\text { A manipulation intrusion directed at ID } 0 \times 5 \mathrm{~d} 7 \text {. Over the course of 50s the first two bytes of each relevant message were } \\
\text { set to zero, while the rest of the payload remains unchanged. This part of the message represents to speedometer value. }\end{array}$ \\
\hline U/RPM_LEFT & $\begin{array}{l}\text { A manipulation intrusion directed at ID } 0 x 29 a \text {. Over the course of 50s the third and fourth byte of each relevant message } \\
\text { is set to zero, while the rest of the payload remains unchanged. These specific bytes represent the revolutions per minute } \\
\text { (RPM) of the left frontal wheel. }\end{array}$ \\
\hline HCRL/Gear, & $\begin{array}{l}\text { Spoofing attacks from the HCRL data set, where every millisecond messages related to RPM/gear information are injected. } \\
\text { The HCRL data set does not synthetically introduce messages after recording, but provides a record of an intrusion from } \\
\text { a running vehicle. }\end{array}$ \\
\hline Model_X & $\begin{array}{l}\text { A synthetic, randomized DoS attack, merged into the log file recorded from a Tesla Model X similar to U/Fuzzy. This test } \\
\text { is primarily used to validate whether the genetic programming approach is transferable to other vehicles data and not } \\
\text { tested with comparable approaches. }\end{array}$ \\
\hline
\end{tabular}

in checkpoints that can be loaded and further trained without any problems with data collected during everyday usage of the vehicle. The approach was fully implemented in python 3 using the DEAP framework [9] for evolutionary computation.

\section{RESULTS}

An important goal of this work is the comparison of GP as an approach to in-vehicle IDS to similar methods proposed. For this purpose, we have chosen ANN as an example of standard machinelearning techniques, as well as $\mathrm{CF}$ [4] as a rule-based approach utilizing FC on BA payload values. In machine learning, it is especially important to observe model results not only during training, but also during evaluation under real-life scenarios with unknown data. Therefore, we did not evaluate the same intrusion scenarios used during training, but created a high variation of test data sets to show that the models are not only able to classify their specifically trained intrusion settings correctly but potentially detect unknown threats.

Training. All approaches have been trained on the same intrusion data, with the exception of the CF approach that does not require the data to contain intrusions. The training log contains a multitude of small randomized DoS intruders that contain either completely random payloads or copy the payload of the precedent message from the same ID and randomize multiple bytes. These intruders are not modelled after any real intrusion scenario, but only to provide randomized data. Thus no intrusion data from actual recorded incidents is required for training. For the GP approach, there is one additional model trained for data using FC alignments. Both, the CF and the ANN approach, are only trained on BA data. All approaches were trained on the same server.

In training the GP approach, BA data resulted in the computation of the smallest individuals with a size of 402 nodes for ID $0 \times 29 a$ and 383 for ID $0 \times 5 \mathrm{~d} 7$. Training on the data using FC alignments does not only show a significant increase in size, but also a slight decrease in maximum fitness and in the case for ID $0 \times 29 a$ a significant increase in training time. For the ANN approach, input data was split in an $80 / 20$ ratio into a training and validation set. The predicted accuracy of the model is greater than $99 \%$ for both IDs on the training data as well as on the validation data. The networks trained for both IDs contain 1,101 trainable parameters each.

The training time for GP is in its current implementation significantly higher compared to ANN and CF. GP model computation took about one hour of training time, where ANN only used approximately 10 minutes and CF under one minute. It should be noted that the implementation of the GP approach was not performanceoptimized. The training time does not affect the classification time during actual usage of the models and is typically not performed on embedded edge devices.

Intrusion Detection. The results for the intrusion detection performance are shown in Table 2. The statistics are derived from the number of true positive (TP), false positive (FP), true negative (TN) and false negative (FN) messages, whereby intrusion messages are labelled positives and non-intrusion messages are labelled negatives. Positive Predictive Value or Precision $(P P V=T P /(T P+F P))$ is the portion of true positives within all positive calls. True Positive Rate $(T P R=T P /(T P+F N))$ denotes the portion of positive values that are correctly classified as true positive. Accuracy $(A C C=(T P+T N) /(T P+T N+F P+F N))$ is the portion of correctly classified messages, regardless whether they are positive or negative.

In the GP approach, the U/Fuzzy intrusion showed good results in both models, with an increased number of FP for the FC model. The U/DoS scenario was not considered in the training data and therefore the models perform worse in most cases. The targeted U/RPM_LEFT was detected with a TPR of $100 \%$ using BA. The respective field classification model was only able to detect $\sim 11 \%$ of intrusion messages. The U/SPEED intrusion for ID $0 \times 5 \mathrm{~d} 7$ could 
Table 2: Intrusion detection results w.r.t. synthetic log files based on Renault ZOE data for ID $0 \times 29 a$ marked by dash and for ID 0x5d7 marked by double dash for genetic programming (GP) (with alignments by field classification (FC) and byte-alignments (BA)) as well as artificial neural network (ANN) and characteristic functions (CF) both using BA. Model_X and the HCRL datasets were only tested for GP.

\begin{tabular}{|c|c|c|c|c|c|c|c|c|c|c|c|c|}
\hline \multirow[b]{2}{*}{ Log file } & \multicolumn{3}{|c|}{ GP-FC } & \multicolumn{3}{|c|}{ GP-BA } & \multicolumn{3}{|c|}{ ANN-BA } & \multicolumn{3}{|c|}{ CF-BA } \\
\hline & $\mathrm{ACC}$ & PPV & TPR & $\mathrm{ACC}$ & PPV & TPR & $A C C$ & PPV & TPR & $\mathrm{ACC}$ & PPV & TPR \\
\hline U/Fuzzy' & .952 & .769 & .994 & $\mid .990$ & .982 & .958 & .999 & .999 & 1 & 1 & 1 & 1 \\
\hline U/Dos' & .921 & .946 & .528 & .905 & .625 & 1 & .941 & .986 & .634 & .999 & .997 & .997 \\
\hline U/RPM_LEFT' & .914 & .800 & .115 & .998 & .980 & 1 & .984 & .998 & .826 & .839 & .366 & 1 \\
\hline U/Fuzzy” & .981 & .963 & .999 & \begin{tabular}{|l|}
.995 \\
$\mid$
\end{tabular} & .997 & .992 & .999 & 1 & .999 & $\mid 1$ & 1 & 1 \\
\hline U/DoS" & .499 & .201 & .012 & .904 & .834 & 1 & .942 & .986 & 1 & 1 & 1 & 1 \\
\hline U/SPEED” & .957 & .716 & .894 & .906 & $\mathbf{0}$ & $\mathbf{0}$ & .906 & - & $\mathbf{0}$ & .186 & $\mathbf{0}$ & $\mathbf{0}$ \\
\hline Model_X & 1 & 1 & 1 & .998 & .998 & .994 & \multirow{3}{*}{\multicolumn{3}{|c|}{ Not measured }} & \multirow{3}{*}{\multicolumn{3}{|c|}{ Not measured }} \\
\hline HCRL/RPM & .901 & .892 & 1 & .910 & .891 & 1 & & & & & & \\
\hline HCRL/Gear & .912 & .901 & 1 & .914 & .897 & 1 & & & & & & \\
\hline
\end{tabular}

only be detected using the field classification model, while the BA model did not detect any of the messages.

The intrusion mixed into the Model_X data was detected perfectly for FC alignments and with only a small number of false predictions for the BA data. It is to be noted that the model used for the Model_X DoS intrusion was trained on a separate training set, where similar random artificial messages used for the main training log were mixed into the Model_X $\log$ file. HCRL/Gear and HCRL/RPM were both detected with a perfect TPR, but a slightly higher number for FP. This is due to the high number of introduced intrusion messages in the data set, which are all relatively similar to the original non-intrusion messages. The targeted intrusion for the U/RPM_LEFT field did yield a PPV of $\sim 99.8 \%$ with a TPR of only $\sim 82 \%$ and therefore can be considered to be successfully detected. For the respective targeted intrusion on the U/SPEED field for ID $0 \times 5 \mathrm{~d} 7$ no intrusion message was correctly classified.

With the CF approach, all intrusion messages from the targeted RPM_LEFT test log were correctly classified, but with a high proportion of FP. For ID $0 \times 5 d 7$ no intrusion messages from the targeted SPEED intrusion were correctly classified. The results also show a significant amount of FP messages with an overall accuracy of $\sim 18 \%$.

For an in-vehicle intrusion detection approach not only the accuracy of the prediction, but also the performance on edge components within the vehicle is very important. Our test used a Raspberry Pi 3 Model B to make the results comparable to other recently published work that uses the same platform, e.g. [31]. Classification through Tensorflow ANN is computation-intensive and requires most time to predict the class of one message, with only $\sim 140$ messages per second. GP performs on average about six times faster and $\mathrm{CF}$ are on average over ten times faster than ANN.

\section{DISCUSSION}

In this section, we discuss not only on how GP performed on invehicle intrusion detection, but also on the impact of prior FC and how this approach compares to the competing methods examined.
Genetic Programming Intrusion Detection. For the ID 0x29a, BA and FC models were able to successfully classify messages from a different recording on the same vehicle, while they could not achieve $100 \%$ accuracy with a clean $\log$ file. This might be solved through additional training and a higher focus on valid log detection through adjustment of the factor $f$ in fitness evaluation. FC showed a higher number of FP in a different randomized DoS intrusion and a low TPR in the zero value DoS scenarios. This might also indicate a biased training on static or low variant fields. The targeted manipulation of the Rpm_Left value could not be detected, which further strengthens the previous assumption. The BA model, however, was able to completely recognize said targeted intrusion as well as the zero value $D o S$. This heavily indicates that more complex correlations between the different field values have been discovered. For the ID $0 \times 5 \mathrm{~d} 7$, the U/SPEED intrusion was detected with 90\% TPR using the FC model. This intrusion is especially important because it utilizes valid values for the Speed sensor that can only be detected through observation of the changes in the fine distance field. No changes in the latter field imply a standing vehicle with Speed of 0 , whereas changes imply a moving vehicle with Speed over 0 . This complex intrusion could not be detected with the BA model. The results for the Model_X and HCRL intrusion scenarios show that this method also works well on data from other vehicles. For further development, a combination of both methods could be beneficial. Multiple models trained with different alignments, different sets of primitives and terminals, as well as varying training parameters could be combined through the random forest [2] method of combining decision trees. On their own, one tree might focus too much on a single characteristic of the data or grow indefinitely large to incorporate every characteristic and is therefore prone to errors. For a real-life scenario the heuristics for fitness in GP have to be further adjusted to punish FP messages more severely, due to slightly increased FP rate compared to the other approaches.

Comparison of Methods. In general, the ANN approach achieved similar results as the GP approach for both IDs. However, each 
method displays specific strengths for certain training sets. For most intrusions, accuracy of the ANN was slightly higher, with only the U/RPM_LEFT intrusion being classified significantly better by the GP model and the U/SPEED intrusion that could not be classified at all by ANN. Results show that models constructed with GP are better at discovering correlations between fields, especially if changes between fields are required for successful classification. In contrast, classification accuracy for the U/DoS and U/Fuzzy intrusion is higher for ANN than for the GP approach which indicates a superiority of ANN in training to check numeric values for ranges or fixed values. The $\mathrm{CF}$ approach excels in classifying randomized values when values are out of the usual range or simply zero value. In the U/SPEED intrusion test the algorithm only discovered an anomalous change in data after the intrusion was finished and subsequently flagged every message afterwards falsely as an intrusion. This shows an potential problem with the algorithm already discussed by Chevalier et al.[4].

Suggestions for further improvements. A combined approach of GP could prove to be promising, utilizing multiple decision trees in parallel, with some of them trained on data with FC alignments and others without. This hybrid approach is currently being evaluated by us. Also, additional operators for the generation of the GP model, such as fuzzy comparisons or arithmetic operators, might be interesting to be evaluated. The capabilities to detect complex relations might make our GP approach interesting to be used in combination with flow-based methods, as well as with approaches such as the CF method that focus on exact value ranges. In summary, a combination of an approach such as CF with our GP approach might be highly beneficial. The $\mathrm{CF}$ approach is convincing in finding values out of the usual ranges, but has difficulties in finding inconsistencies in data that is spread over multiple fields or finding correlations within data in general. GP has proved capable of finding complex relationships between different fields, but in the current implementation has shown difficulties in finding individual anomalous values outside certain ranges. Using both methods in sequence or multiple models in parallel, with a majority voting mechanism for classification approach might be interesting to test, because this may combine the benefits of both approaches, while minimizing their weaknesses.

\section{CONCLUSION}

The aim of this work was to improve the detection of targeted attacks on the CAN bus in order to reduce malicious effects on safety-relevant vehicle components. In particular, the suitability of GP for in-vehicle IDS has been evaluated. We took into account Sommer and Paxson's [30] findings that insight is far more important than numerical results in this area, and we carefully analyzed what it can detect and why and what it cannot and why not.

In conclusion, our research found that decision trees trained with the proposed GP approach show good results in detecting complex intrusions based on relationships between different fields. In the case of DoS attacks, the method depends heavily on the training data. Randomized DoS intrusions could be detected with a higher accuracy than DoS attacks with a value of zero, which were not previously observed in training. This approach has only proven to be inferior to other methods in this area if it is necessary to consider specific value ranges. FC of the payload prior to training has been found helpful in some cases, while unclassified BA data has provided better results in others. The results of GP show only a slight improvement in classification accuracy over ANN, but show a significant advantage in classification performance, which is important for use in areas with limited resources.

\section{ACKNOWLEDGMENTS}

This research work has been partly funded by the German Federal Ministry of Education and Research (BMBF) and the Hessen State Ministry for Higher Education, Research and the Arts within their joint support of the National Research Center for Applied Cybersecurity ATHENE and by the BMBF project VITAF (ID 16KIS0835). Additionally, the project leading to this application has received funding from the European Union's Horizon 2020 research and innovation programme under grant agreement No 883135 .

\section{REFERENCES}

[1] Omar Y Al-Jarrah, Carsten Maple, Mehrdad Dianati, David Oxtoby, and Alex Mouzakitis. 2019. Intrusion Detection Systems for Intra-Vehicle Networks: A Review. IEEE Access 7 (2019), 21266-21289.

[2] Leo Breiman. 2001. Random Forests. Machine Learning 45, 1 (01 Oct 2001), 5-32. https://doi.org/10.1023/A:1010933404324

[3] Susan M Bridges, Rayford B Vaughn, et al. 2000. Fuzzy data mining and genetic algorithms applied to intrusion detection. In Proceedings of 12th Annual Canadian Information Technology Security Symposium. 109-122.

[4] Yannick Chevalier, Roland Rieke, Florian Fenzl, Andrey Chechulin, and Igor V. Kotenko. 2020. ECU-Secure: Characteristic Functions for In-Vehicle Intrusion Detection. In Intelligent Distributed Computing XIII (Studies in Computational Intelligence, Vol. 868), Igor V. Kotenko, Costin Badica, Vasily Desnitsky, Didier El Baz, and Mirjana Ivanovic (Eds.). Springer, Cham, 495-504. https://doi.org/10. 1007/978-3-030-32258-8_58

[5] M Crosbie and E Spafford. 1995. Applying Genetic Algorithms to Intrusion Detection. In Proceedings of the AAAI.

[6] Marco Di Natale, Haibo Zeng, Paolo Giusto, and Arkadeb Ghosal. 2012. Understanding and using the controller area network communication protocol: theory and practice. Springer Science \& Business Media.

[7] ENISA. 2016. Cyber Security and Resilience of smart cars. Technical Report. ENISA. https://doi.org/10.2824/87614

[8] Bob Fisch and Jeroen Meijer. 2016-. CanZE: Take a closer look at your ZOE. https://github.com/fesch/CanZE

[9] Félix-Antoine Fortin, François-Michel De Rainville, Marc-André Gardner, Marc Parizeau, and Christian Gagné. 2012. DEAP: Evolutionary Algorithms Made Easy. Journal of Machine Learning Research 13 (jul 2012), 2171-2175.

[10] Sébastien Gay, Laurent Sartran, and Jean-Philippe Vasseur. 2019. Specializing unsupervised anomaly detection systems using genetic programming. (26 Feb 2019). , US Patent 10,218,729.

[11] Anup Goyal and Chetan Kumar. 2008. GA-NIDS: a genetic algorithm based network intrusion detection system. Northwestern university (2008).

[12] Hacking and Countermeasure Research Lab (HCRL). 2018. Car-Hacking Dataset for the intrusion detection. http://ocslab.hksecurity.net/Datasets/CAN-intrusiondataset. [Online; accessed 28-Jun-2018].

[13] Pohlheim Hartmut. 2004. Evolutionary Algorithms: Overview, Methods and Operators. GEATbx version 3 (2004).

[14] Tobias Islinger, Yasuhiro Mori, Jennifer Neumüller, Martin Prisching, and Robert Schmidt. 2017. Autosar SecOC for CAN FD. https://cannewsletter.org/engineering/engineering-miscellaneous/170306_autosar-secocfor-can-fd_denso

[15] Karl Koscher, Alexei Czeskis, Franziska Roesner, Shwetak Patel, Tadayoshi Kohno, Stephen Checkoway, Damon McCoy, Brian Kantor, Danny Anderson, Hovav Shacham, et al. 2010. Experimental security analysis of a modern automobile. In 2010 IEEE Symposium on Security and Privacy. IEEE, 447-462.

[16] John R Koza. 1997. Genetic programming. (1997).

[17] Wei Lu and Issa Traore. 2004. Detecting new forms of network intrusion using genetic programming. Computational intelligence 20, 3 (2004), 475-494.

[18] Parry Gowher Majeed and Santosh Kumar. 2014. Genetic algorithms in intrusion detection systems: A survey. International fournal of Innovation and Applied Studies 5, 3 (2014), 233.

[19] Mirco Marchetti and Dario Stabili. 2017. Anomaly detection of CAN bus messages through analysis of ID sequences. In 2017 IEEE Intelligent Vehicles Symposium (IV). IEEE, 1577-1583. 
[20] Moti Markovitz and Avishai Wool. 2017. Field classification, modeling and anomaly detection in unknown CAN bus networks. Vehicular Communications 9 (2017), 43-52.

[21] Brad L Miller, David E Goldberg, et al. 1995. Genetic algorithms, tournament selection, and the effects of noise. Complex systems 9, 3 (1995), 193-212.

[22] Charlie Miller and Chris Valasek. 2013. Adventures in automotive networks and control units. Def Con 21 (2013), 260-264.

[23] Charlie Miller and Chris Valasek. 2015. Remote exploitation of an unaltered passenger vehicle. Black Hat USA 2015 (2015), 91.

[24] David J Montana. 1995. Strongly typed genetic programming. Evolutionary computation 3, 2 (1995), 199-230.

[25] Controller Area Network. 1991. Specification-Version 2.0. Bosch GmbH (1991).

[26] Sen Nie, Ling Liu, and Yuefeng Du. 2017. Free-fall: hacking tesla from wireless to CAN bus. Briefing, Black Hat USA (2017), 1-16.

[27] Riccardo Poli, Mathew Salvaris, and Caterina Cinel. 2011. Evolution of a braincomputer interface mouse via genetic programming. In European Conference on Genetic Programming. Springer, 203-214.

[28] T. Rosenstatter, C. Sandberg, and T. Olovsson. 2019. Extending AUTOSAR's Counter-Based Solution for Freshness of Authenticated Messages in Vehicles. In 2019 IEEE 24th Pacific Rim International Symposium on Dependable Computing (PRDC). 1-109.

[29] Eunbi Seo, Hyun Min Song, and Huy Kang Kim. 2018. Gids: Gan based intrusion detection system for in-vehicle network. In 2018 16th Annual Conference on Privacy, Security and Trust (PST). IEEE, 1-6.
[30] R. Sommer and V. Paxson. 2010. Outside the Closed World: On Using Machine Learning for Network Intrusion Detection. In 2010 IEEE Symposium on Security and Privacy. IEEE, 305-316. https://doi.org/10.1109/SP.2010.25

[31] Hyun Min Song, Jiyoung Woo, and Huy Kang Kim. 2020. In-vehicle network intrusion detection using deep convolutional neural network. Vehicular Communications 21 (2020), 100198.

[32] Ivan Studnia, Vincent Nicomette, Eric Alata, Yves Deswarte, Mohamed Kaâniche, and Youssef Laarouchi. 2013. Security of embedded automotive networks: state of the art and a research proposal. In SAFECOMP 2013 - Workshop CARS of the 32nd International Conference on Computer Safety, Reliability and Security, Matthieu ROY (Ed.).

[33] Adrian Taylor. 2017. Anomaly-based detection of malicious activity in in-vehicle networks. Ph.D. Dissertation. Université d'Ottawa/University of Ottawa.

[34] Adrian Taylor, Sylvain Leblanc, and Nathalie Japkowicz. 2018. Probing the Limits of Anomaly Detectors for Automobiles with a Cyberattack Framework. IEEE Intelligent Systems 33, 2 (2018), 54-62.

[35] Miki E Verma, Michael D Iannacone, Robert A Bridges, Samuel C Hollifield, Bill Kay, and Frank L Combs. 2020. ROAD: The Real ORNL Automotive Dynamometer Controller Area Network Intrusion Detection Dataset (with a comprehensive CAN IDS dataset survey \& guide). arXiv preprint arXiv:2012.14600 (2020).

[36] Daniel Zelle, Roland Rieke, Christian Plappert, Christoph Krauß, Dmitry Levshun, and Andrey Chechulin. 2020. SEPAD-Security Evaluation Platform for Autonomous Driving. In 2020 28th Euromicro International Conference on Parallel, Distributed and Network-Based Processing (PDP). IEEE, 413-420. https: //doi.org/10.1109/PDP50117.2020.00070 